\title{
Pathophysiological correlations between fundus fluorescein angiography and optical coherence tomography results in patients with idiopathic epiretinal membranes
}

\author{
JU LIU ${ }^{1-3}$, YONG QIAN $^{3}$, SHUNNAN YANG $^{4}$, LI YAN $^{3}$, YI WANG $^{1,2}$, MENG GAO $^{1,2}$,

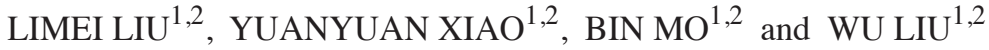 \\ ${ }^{1}$ Department of Ophthalmology, Beijing Tongren Eye Center, Beijing Tongren Hospital; ${ }^{2}$ Beijing Ophthalmology and Visual \\ Sciences Key Laboratory; ${ }^{3}$ Department of Ophthalmology, Beijing Electric Power Hospital, Capital Medical University, \\ Beijing 100073, P.R. China; ${ }^{4}$ Vision Performance Institute, Pacific University College of Optometry, \\ Forest Grove, OR 97116, USA
}

Received February 20, 2017; Accepted August 1, 2017

DOI: $10.3892 / \mathrm{etm} .2017 .5330$

\begin{abstract}
The aim of the current study was to determine the association between fundus fluorescein angiography (FFA) image results, optical coherence tomography (OCT) imaging results and visual functions in patients with idiopathic epiretinal membranes (ERMs). A total of 80 eyes from 40 patients diagnosed with ERM were analyzed. Best-corrected visual acuity (BCVA) and metamorphopsia were measured using the Early Treatment Diabetic Retinopathy Study eye chart and M-charts, respectively. Macular thickness and volume were determined using OCT. Macular vascular leakage and distortion, and foveal avascular zone (FAZ) diameters were assessed using FFA. BCVA and M-chart results confirmed macular degeneration in the affected eyes. The area of macular vascular leakage was positively correlated with macular volume $(\mathrm{r}=0.50 ; \mathrm{P}=0.001)$. The ratio of $\mathrm{FAZ}$ diameters was negatively correlated with central macular thickness $(\mathrm{r}=-0.41$; $\mathrm{P}=0.008$ ). The grade of macular vascular distortion was positively correlated with macular thickness (all $\mathrm{r} \geq 0.47 ; \mathrm{P}<0.01$ ) and volume $(\mathrm{r}=0.53 ; \mathrm{P}<0.001)$. The results of the current study demonstrated that FFA may effectively identify the degree of changes in retinal macular vasculature. Vascular distortion, measured by FFA, may reflect changes in macular structure and visual functions. These results suggest a potential application of FFA in the pre- and post-surgical evaluation of patients with ERMs.
\end{abstract}

Correspondence to: Dr Wu Liu, Department of Ophthalmology, Beijing Tongren Eye Center, Beijing Tongren Hospital, Capital Medical University, 1 Dongjiaominxiang Road, Beijing 100730, P.R. China

E-mail: liuwu2366@126.com

Key words: fundus fluorescein angiography, epiretinal membrane, retinal vasculature, optical coherence tomography

\section{Introduction}

Idiopathic epiretinal membranes (ERMs) are macular disorders frequently diagnosed in the elderly and have an incidence of $2-20 \%$ (1). An ERM is a semi-translucent, glial and fibrocellular proliferative membrane (2). It usually develops on the surface of the internal limiting membrane, particularly on the macular surface, following partial or complete posterior vitreous detachment. Idiopathic ERM formation is a primary disease, rather than a disease secondary to trauma, inflammation, surgery, photocoagulation or cryotherapy.

Contraction or shrinkage of proliferative membranes may create irregular folds in the membrane itself, and exert anteroposterior and/or tangential tractional forces on the retina and retinal vasculature. The anteroposterior force produces vertical traction and increases retinal thickness; the tangential force drags the superficial retinal layers away from their original position, causing deformation and displacement of the retina. ERMs also alter the morphology, location and permeability of the retinal vasculature. Morphological abnormalities include the straightening and/or curling of retinal blood vessels and the irregularity and shrinkage of the foveal avascular zone (FAZ). Several studies have demonstrated that retinal vessels and the retina itself may become displaced due to the tractional forces caused by the ERM $(3,4)$.

Fundus fluorescein angiography (FFA) is one of the optimal methods used to observe retinal vascular morphology and the degree of vascular leakage. Due to the almost transparent nature of the proliferative membrane, FFA allows clear observation of the deformed retinal vasculature under the ERM and occasionally, the straightening, displacement and contraction of the FAZ. Alterations in macular structure, primarily in macular thickness and vasculature, cause visual problems in patients with ERMs $(4,5)$. Although the effects of macular structural alteration on visual function have been investigated using ocular coherence topography (OCT), few studies have documented the effects of retinal vasculature alteration (6-9). Furthermore, to the best of our knowledge, there have been no studies discussing the potential impact 
of retinal vascular changes on retinal structure and visual function. Advancements in FFA technology may allow the reliable measurement of vascular changes and facilitate future studies into these issues. The present study therefore aimed to determine the pathophysiological mechanisms underlying the formation and evolving severity of ERMs. FFA measured the area of macular vascular leakage, grade of retinal vascular distortion and contraction of the FAZ. FFA imaging results were correlated with OCT measurements and visual functions.

\section{Patients and methods}

Patients. Patients were recruited from outpatient clinics at Beijing Tongren Hospital (Beijing, China). The inclusion criteria included patients with a diagnosis of symptomatic idiopathic ERMs in one eye using OCT and an ophthalmoscope. Patients were excluded if they had secondary ERMs, ERMs with lamellar or full-thickness macular holes, systemic or ocular conditions that may affect the macular anatomy and the results of the FFA, including cystoid macular edema, bleeding and fluorescein pooling.

A total of 40 adults with ERM were enrolled in the current study and 80 eyes were studied between September 2013 and March 2014 at Beijing Tongren Hospital. Informed consent was obtained from all participants and the current study was approved by the Ethics Committee at Beijing Tongren Hospital.

Measurements of visual acuity and metamorphopsia. All recruited patients underwent a comprehensive ophthalmic examination. Best-corrected visual acuity (BCVA) of the affected eyes was measured at a distance of $4 \mathrm{~m}$ using the Early Treatment Diabetic Retinopathy Study (ETDRS) eye chart (Precision Vision, Woodstock, IL, USA) and quantified as the logarithmically transformed score of the minimal angle of resolution (logMAR). The ETDRS chart included logMAR labels for each letter size andscring was performed by giving equal credit (0.02 $\log$ MAR units) for each extra letter read correctly (10).

Metamorphopsia was determined using M-charts (Inami \& Co., Ltd., Tokyo, Japan), which consist of 19 dotted lines with dot intervals of between 0.2 (fine) and 2.0 (coarse) visual angles. If patients recognized one of the dotted lines as straight, its visual angle between dots was considered to be their metamorphopsia score (11). As dot intervals were changed from fine to coarse, a decrease in the severity of metamorphopsia was noted. The examination distance for every patient was $30 \mathrm{~cm}$ with the best refractive correction. Each patient underwent three tests with the vertical and horizontal M-charts and the average of the three scores was recorded (12).

Measurements of retinal vascular leakage, distortion and FAZ by FFA. FFAs were performed on all affected eyes using the Heidelberg Retina Angiograph (Heidelberg Engineering, Ltd., Heidelberg, Germany), according to the manufacturer's protocol. A blinded examiner analyzed the angiograms to determine the area of retinal vascular leakage, as defined by hyper-fluorescence on the angiogram visible $10 \mathrm{~min}$ after fluorescein injection. The examiner also graded the distortion of macular vasculature and the contraction of the FAZ during the venous phase.

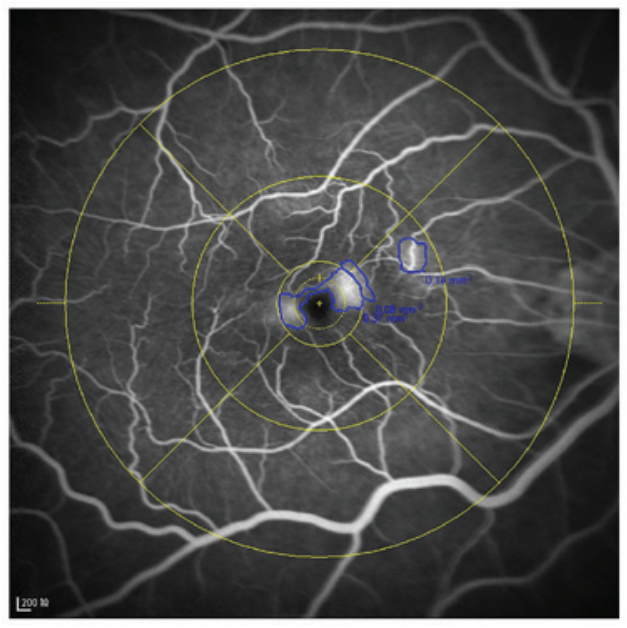

Figure 1. Measurement of macular vascular leakage by fundus fluorescein angiography. The outline of leakage area in the macula was manually drawn (blue lines) inside the Early Treatment Diabetic Retinopathy Study grid (yellow lines; diameter, $6 \mathrm{~mm}$ ). Scale bar, $200 \mu \mathrm{m}$.

Leakages in the FFA images were quantified by manually outlining the leakage area within the ETDRS grid (diameter, $6 \mathrm{~mm}$; Fig. 1), then computing the total area of the leakage into the software built into the Heidelberg Retinal Angiograph. Macular leakage was graded on a scale of 1-4 (1, no leakage; 2 , vascular leakage outside the central $1 \mathrm{~mm}$ area and no leakage inside it; 3 , vascular leakage within the central $1 \mathrm{~mm}$ area but no leakage outside of it; and 4 , vascular leakage inside and outside of the central $1 \mathrm{~mm}$ area), based on the method described by Maguire et al (9) (Fig. 2).

Retinal vascular distortion (Fig. 3) was graded on a scale of 0-4 based on Maguire et al method (9); 0 , no evidence of vascular straightening or distortion; 1, straightening and/or distortion within one $90^{\circ}$ quadrant relative to the fovea; 2 , straightening and/or distortion involving two quadrants; 3 , vascular straightening and/or distortion involving three quadrants; and 4, straightening and/or distortion involving all four quadrants). Straightening also included loss of normal vascular tortuosity.

Minimum diameters of FAZ were measured on the affected and fellow eyes by manually drawing the borderline of the FAZ and measuring their diameters using a digital scale. To control for individual variability in FAZ measurements, the ratio of the FAZ in the ERM eye to the FAZ in the fellow eye was calculated. The calculated ratio indicated the degree of contraction of the central zone and the surrounding capillary network.

OCT measurements of macular thickness and volume. Macular thickness and volume were measured using Cirrus HD-October 5000 (Carl Zeiss AG, Oberkochen, Germany), according to the manufacturer's protocol. The scanning pattern was based on the fast macular map (resolution, 200x200), which measures retinal thickness from the surface of the internal limiting membrane to Bruch's membrane. Mean thickness of the macular areas within the ETDRS grid were measured. The central macular thickness (CMT) was defined as the thickness of the central zone (diameter, $1 \mathrm{~mm}$ ). The inner ring was defined as the circular area surrounding the 

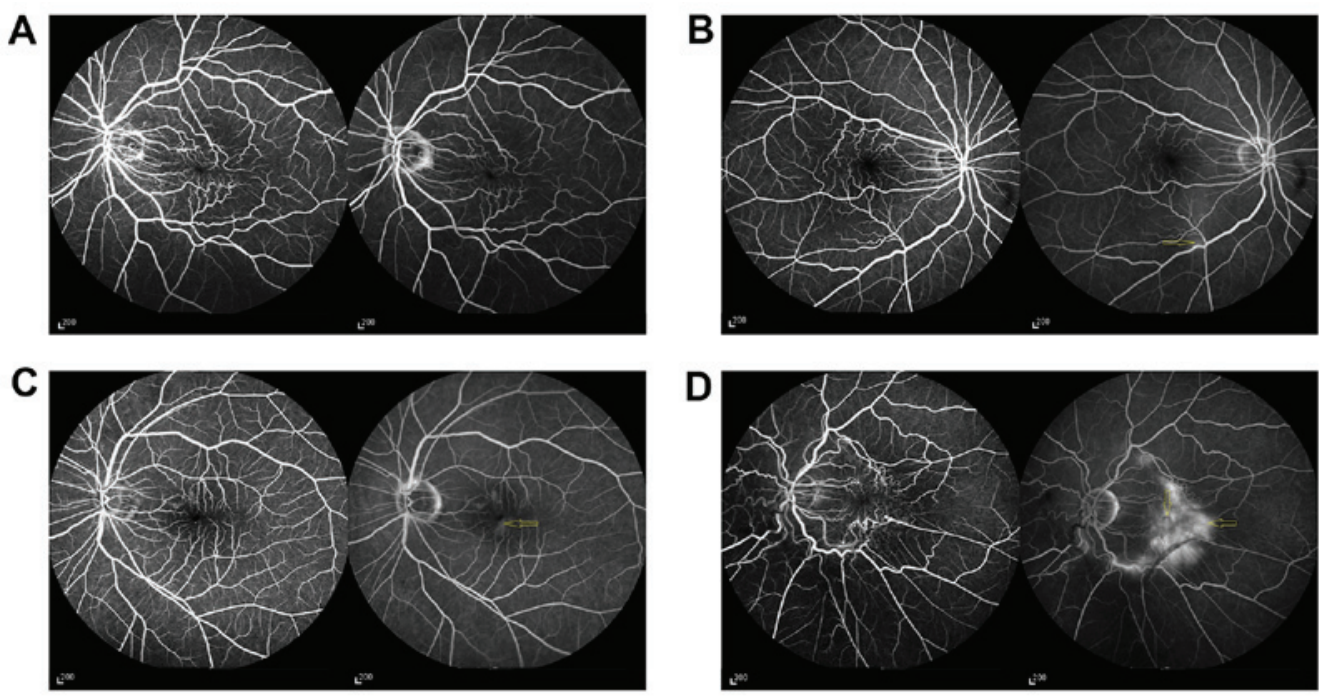

Figure 2. Visual representation of retinal vascular leakage grading. The images on the left refer to arterial phase, images on the right refer to late phase Vascular leakage was graded on a scale of 1-4, as defined by the location of hyper-fluorescence. (A) Grade 1, no vascular leakage. (B) Grade 2, vascular leakage only outside the central $1 \mathrm{~mm}$ area. (C) Grade 3, vascular leakage only within the central $1 \mathrm{~mm}$ area. (D) Grade 4, vascular leakage inside and outside of the central $1 \mathrm{~mm}$ area. Arrows present the location of the leakages. Scale bar, $200 \mu \mathrm{m}$.
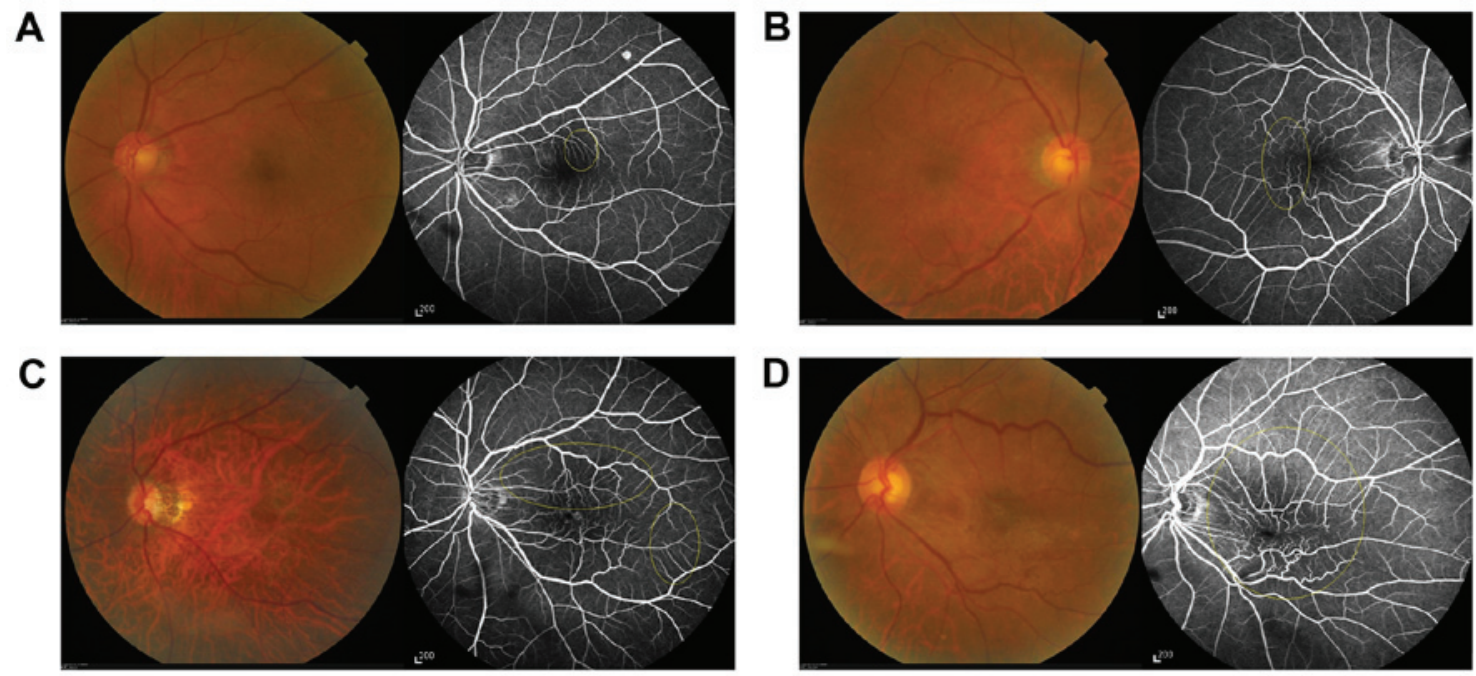

Figure 3. Visual representation of retinal vascular distortion grading. Vascular distortion in fundus fluorescein angiographs was graded on the extent of straightening and/or distortion in the ellipses (yellow circles). (A) Grade 1, straightening and/or vascular tortuosity limited to a $90^{\circ}$ quadrant centered on the fovea. (B) Grade 2, straightening of the retinal vessels within two quadrants. (C) Grade 3, vascular straightening within three quadrants. (D) Grade 4, vascular straightening in all four quadrants. Scale bar, $200 \mu \mathrm{m}$.

CMT (width, $1 \mathrm{~mm}$ ). The outer ring was defined as the circular area surrounding the inner ring (width, $1.5 \mathrm{~mm}$ ). Thickness readings of the four quadrants for each ring were taken and the mean of macular thickness was calculated for the inner and outer rings. The total macular volume was measured and recorded by OCT. For all clinical examinations, examiners were not told the results of the angiogram analysis.

Statistical analysis. BCVA results are presented as $\log$ MAR scores. OCT results are presented as the value of thickness and volume. FFA results are presented as the value of leakage area and grading of vascular image. Pearson's correlation analysis was used to determine the association among the size of leakage area, the ratio of FAZ diameters, and mean macular thickness and volume. Since there were heterogeneous distributions for the grading of vascular distortion (K-S test, $\mathrm{z}=8.6 ; \mathrm{P}=0.035)$ and macular leakage (K-S test, $\mathrm{z}=31.0$; $\mathrm{P}=0.0001)$, the non-parametric Spearman correlation was used to analyze the association between grading of vascular distortion and other factors, including BCVA, M-chart scores, OCT measurements and FFA findings. $\mathrm{P}<0.05$ was considered to indicate a statistically significant difference for two-tailed tests of calculated correlational coefficients. All statistical analysis was performed using SPSS V.24 (IBM Corp., Armonk, NY, USA).

\section{Results}

Symptomatic primary ERMs are more likely to be severe and affect elderly patients. The mean age of the recruited patients 
was 62.3 \pm 8.2 years old (range, 33-78 years), demonstrating that it is primarily the elderly population that is affected by ERMs (data not shown). Of the 40 patients, $77.5 \%$ were female and $47.5 \%$ possessed an ERM in their right eyes. The mean duration of symptoms was $12.4 \pm 13.3$ months (range, 1-60 months).

The results of FFA demonstrated that vascular distortion and leakage were severe (grade $\geq 3$ ) in the majority of patients (Table I). Table I also details the severity of the measurements in the patients by grading the values. The results of BCVA, $\mathrm{M}$-chart and OCT were moderate in the majority of patients.

Vascular leakage and distortion increase with macular thickness and volume, whereas the FAZ ratio decreases as the CMT increases. Table II presents the association between OCT and FFA measurements. The macular leakage area was positively correlated with the thickness of the outer ring in the ETDRS grid (Fig. 4A) and the macular volume obtained with OCT (Fig. 4B). No correlation was identified among the macular leakage area and CMT or inner ring thickness. However, a negative correlation between the ratio of FAZ diameters and CMT was identified (Fig. 5).

Table II also identified positive correlations among the grade of vascular distortion, and the thickness of the macular sections (the central zone, inner ring and outer ring; Fig. 6A) and the macular volume (Fig. 6B). Positive correlations were also identified among the grade of macular leakage, and the macular volume (Fig. 7A) and thicknesses of the inner and outer rings (Fig. 7B), but not the CMT.

Vascular distortions positively correlate with worsening metamorphopsia and visual acuity, which also worsens with centralized thickening of the macula. The correlations between visual functions (BCVA,M-charts score and symptom duration) and OCT/FFA outcomes were analyzed by Spearman's correlation. Table III demonstrated that positive correlations were identified among BCVA and the CMT (Fig. 8A), the thickness of inner ring (Fig. 8B) and the grade of vascular distortion. A positive correlation was identified between the total M-chart score and the grade of vascular distortion (Fig. 9). However, no correlation was identified between symptom duration and imaging results (Table III). In addition, no correlation was identified between vascular distortion and leakage (data not shown).

\section{Discussions}

The present study analyzed changes in the retinal vasculature of patients with ERM and identified a clear correlation between the degree of certain vascular changes based on FFA results and the thickening of the macular retina based on OCT measurements. Specifically, the area and degree of macular leakage was positively correlated with the thickness of the outer ring and the macular volume. In addition, the thickening of the inner and outer rings was positively correlated with the degree of vascular leakage and distortion. It was indicated that the thickness of the central zone was affected by factors other than increased vascular leakage. To the best of our knowledge, the present study is the first known attempt to investigate the vascular changes of the macula using FFA and determine whether these changes were correlated with alterations in visual functions.
Table I. Summary of visual function and imaging results of patients.

A, Distribution of results of visual function

\begin{tabular}{lcl}
\hline Visual function & Frequency & $\%$ \\
\hline BCVA, logMAR score & & \\
(Snellen score, feet) & & \\
$\log M A R \leq 0.3(\leq 20 / 40)$ & 11 & 27.5 \\
$0.3<\operatorname{logMAR}<0.7(20 / 40-20 / 100)$ & 23 & 57.5 \\
$\log$ MAR $\geq 0.7(\geq 20 / 100)$ & 6 & 15 \\
M-chart total scores & & \\
$0 \leq$ score $\leq 1.0$ & 14 & 35 \\
$1.1 \leq$ score $\leq 3.0$ & 19 & 47.5 \\
$3.1 \leq$ score $\leq 4.4$ & 7 & 17.5 \\
\hline
\end{tabular}

B, Distribution of OCT measurements

\begin{tabular}{lcl}
\hline OCT measurements & Frequency & $\%$ \\
\hline $\mathrm{CMT}, \mu \mathrm{m}$ & 5 & \\
$\mathrm{CMT} \leq 400$ & 31 & 12.5 \\
$400<\mathrm{CMT}<600$ & 4 & 77.5 \\
$\mathrm{CMT} \geq 600$ & & 10 \\
$\mathrm{MV}, \mathrm{mm}^{3}$ & 6 & \\
$\mathrm{MV} \leq 11$ & 27 & 67.5 \\
$11<\mathrm{MV}<14$ & 7 & 17.5 \\
$\mathrm{MV} \geq 14$ & & \\
\hline
\end{tabular}

C, Quantified outcomes of FFA

\begin{tabular}{lccc}
\hline FFA outcomes & Mean & SD & Range \\
\hline Area of leakage, $\mathrm{mm}^{2}$ & 5.61 & 8.90 & $0-44.85$ \\
FAZ ratio & 0.42 & 0.20 & $0.14-0.94$ \\
\hline
\end{tabular}

$\mathrm{D}$, Grading results of vascular image

\begin{tabular}{lccccc}
\hline & \multicolumn{5}{c}{ Subjective grading } \\
\cline { 2 - 6 } Vascular image grade & 0 & 1 & 2 & 3 & 4 \\
\hline Distortion, \% & 0 & 10 & 17.5 & 35 & 37.5 \\
Leakage, \% & - & 12.5 & 7.5 & 27.5 & 52.5 \\
\hline
\end{tabular}

BCVA, best-corrected visual acuity; $\log$ MAR, logarithmically transformed score of the minimal angle of resolution; OCT, optical coherence tomography; CMT, central macular thickness; MV, macular volume; FFA, fundus fluorescein angiography; FAZ, foveal avascular zone; SD, standard deviation.

Tractional forces caused by the ERM consist of the anteroposterior force, which thickens the fovea by shifting it towards the center of the vitreous and the tangential force, which thickens the fovea by shifting the peripheral retinal tissue towards the center of the macula. The present study 
Table II. Associations between OCT and FFA measurements.

\begin{tabular}{lcccc}
\hline & \multicolumn{3}{c}{ Thickness } & \\
\cline { 2 - 4 } & Central zone & Inner ring & Outer ring & Macular volume \\
\hline Area of leakage & 0.26 & 0.30 & $0.55^{\mathrm{c}}$ & $0.50^{\mathrm{b}}$ \\
FAZ ratio & $-0.41^{\mathrm{b}}$ & -0.30 & -0.12 & -0.16 \\
Vascular distortion & $0.62^{\mathrm{c}}$ & $0.59^{\mathrm{c}}$ & $0.47^{\mathrm{b}}$ & $0.53^{\mathrm{c}}$ \\
Vascular leakage & 0.29 & $0.32^{\mathrm{a}}$ & $0.50^{\mathrm{b}}$ & $0.45^{\mathrm{b}}$ \\
\hline
\end{tabular}

${ }^{\mathrm{a}} \mathrm{P}<0.05 ;{ }^{\mathrm{b}} \mathrm{P}<0.01$; and ${ }^{\mathrm{c}} \mathrm{P}<0.001$. OCT, optical coherence tomography; FAZ, foveal avascular zone; FFA, fundus fluorescein angiography.
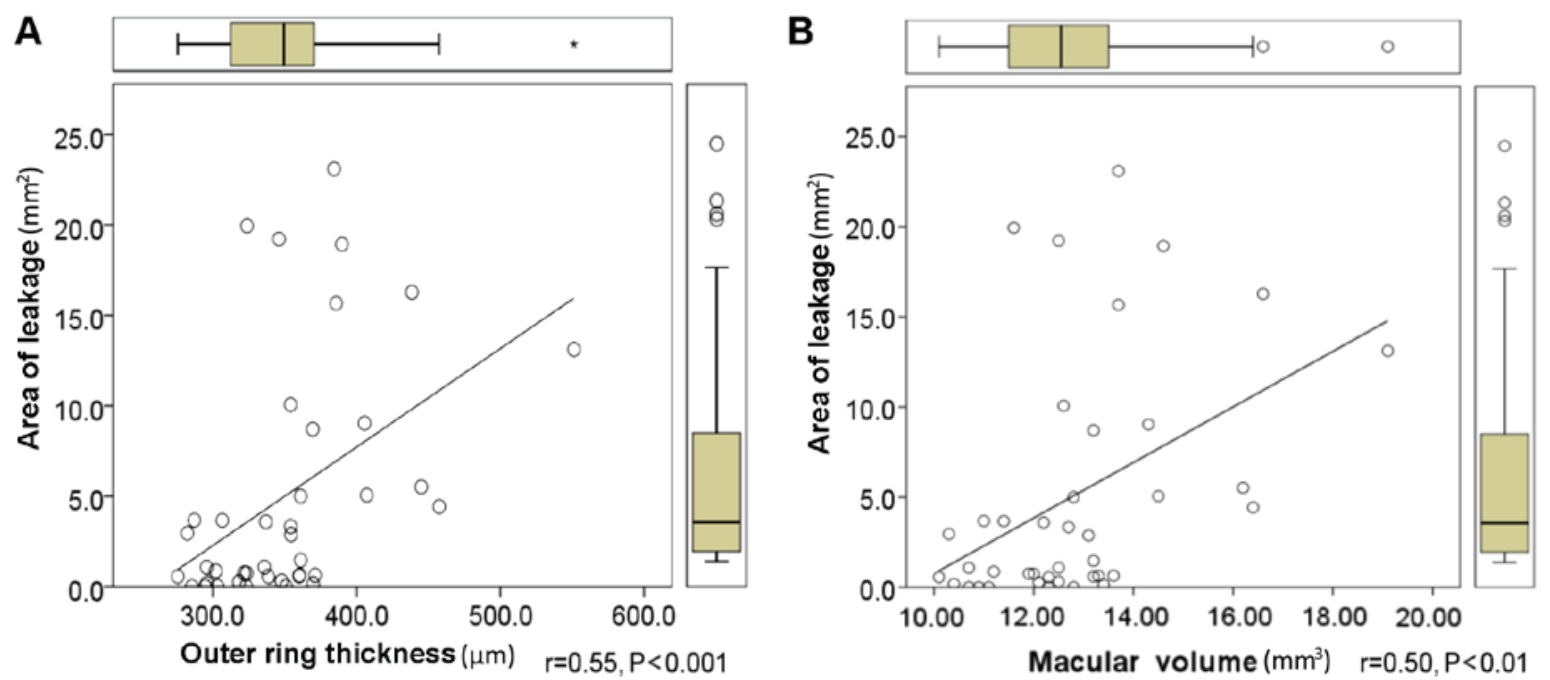

Figure 4. Area of leakage is positively correlated with outer ring thickness and macular volume. The correlation between the area of macular vascular leakage by fundus fluorescein angiography and (A) the outer ring macular thickness by OCT or (B) macular volume by OCT. OCT, optical coherence tomography. Extreme outlier.

identified a negative correlation between the CMT and the ratio of FAZ diameters. A smaller ratio of FAZ diameters may have reflected a greater tangential contraction towards the fovea in the affected eye compared with the fellow eye. The tangential traction force may have caused thickening of the central zone. However, no significant correlation was identified among the ratio of FAZ diameters and the thickness of the inner and outer rings or total macular volume, suggesting that tangential traction primarily affects the CMT.

Tangential traction causes thickening of the fovea and shrinking of the macula, contributing to retinal vascular distortion, which reflects the degree of the twisting and/or straightening of the retinal vessels. Retinal vascular distortion was graded using a semi-quantitative system that does not take into account the distance from the fovea and the severity of vascular distortion. The vascular distortion grade was positively correlated with the thickness of the central zone, inner and outer rings, and macular volume. This suggests that the tangential force from the ERM increases macular thickness and distorts retinal vasculature.

Anteroposterior and tangential forces on the retina may alter vascular permeability (11), and consequently contribute to an increase in macular volume. In the present study,

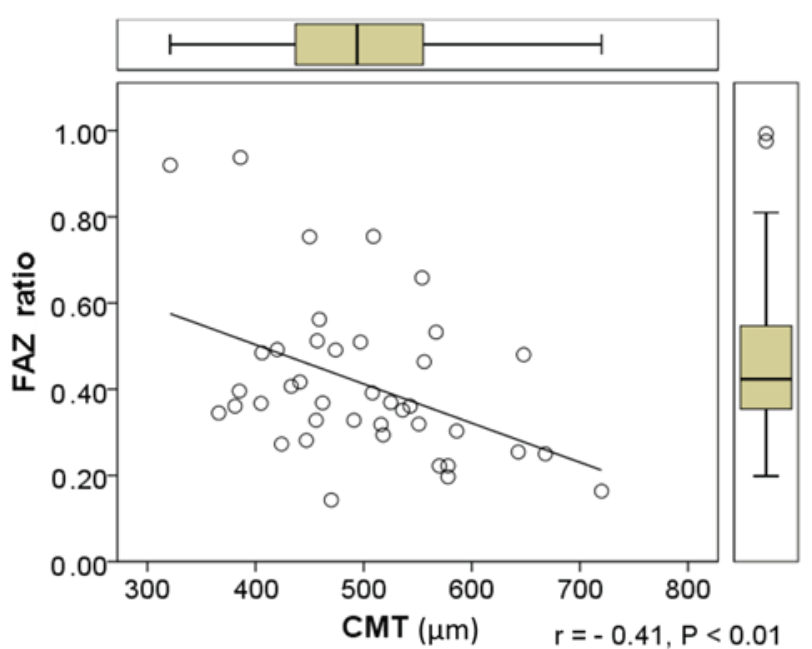

Figure 5. As the central zone thickens, the FAZ ratio decreases. The FAZ and $\mathrm{CMT}$ were measured by fundus fluorescein angiography and optical coherence tomography, respectively. FAZ, foveal avascular zone; CMT, central macular thickness.

the increase in macular volume in patients with ERM was positively correlated with the extent of macular leakage. The 
Table III. Associations between imaging techniques and visual functions.

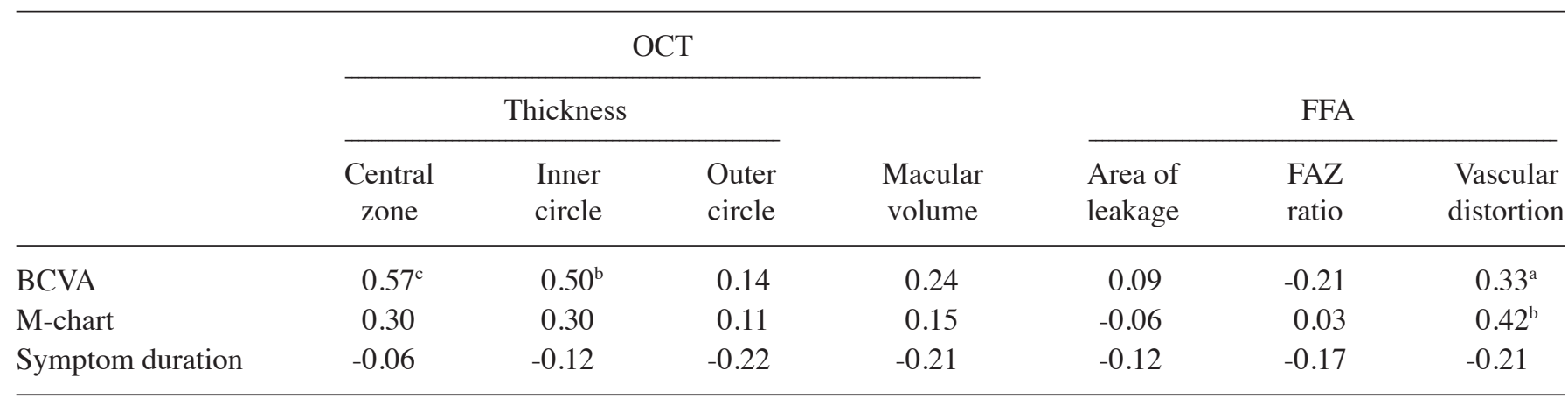

${ }^{\mathrm{a}} \mathrm{P}<0.05 ;{ }^{\mathrm{b}} \mathrm{P}<0.01 ;{ }^{\mathrm{C}} \mathrm{P}<0.001$. BCVA, best-corrected visual acuity; OCT, optical coherence tomography; FAZ, foveal avascular zone; FFA, fundus fluorescein angiography.
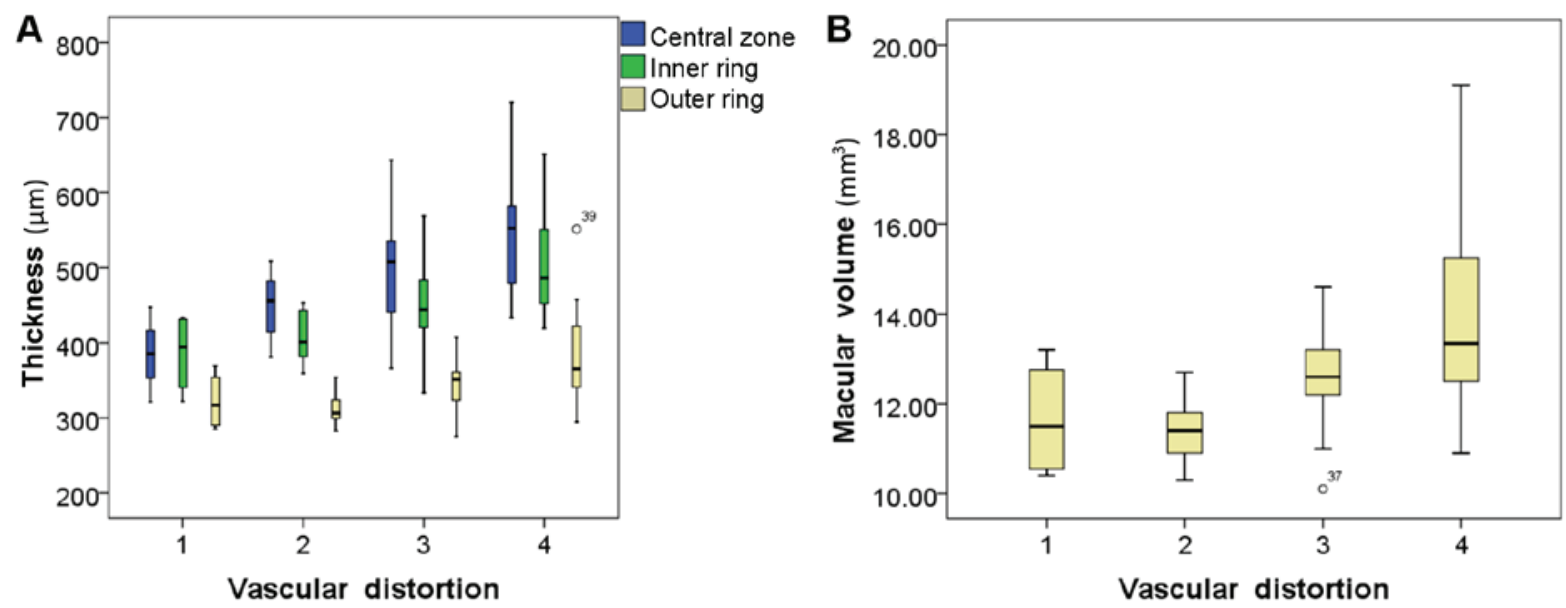

Figure 6. Macular vascular distortion is positively correlated with whole macular thickness and volume. Macular vascular distortion (measured by fundus fluorescein angiography) was positively correlated with (A) whole macular thickness and (B) volume (measured by optical coherence tomography). Circles indicate outliers and are labelled with the subject's serial number.
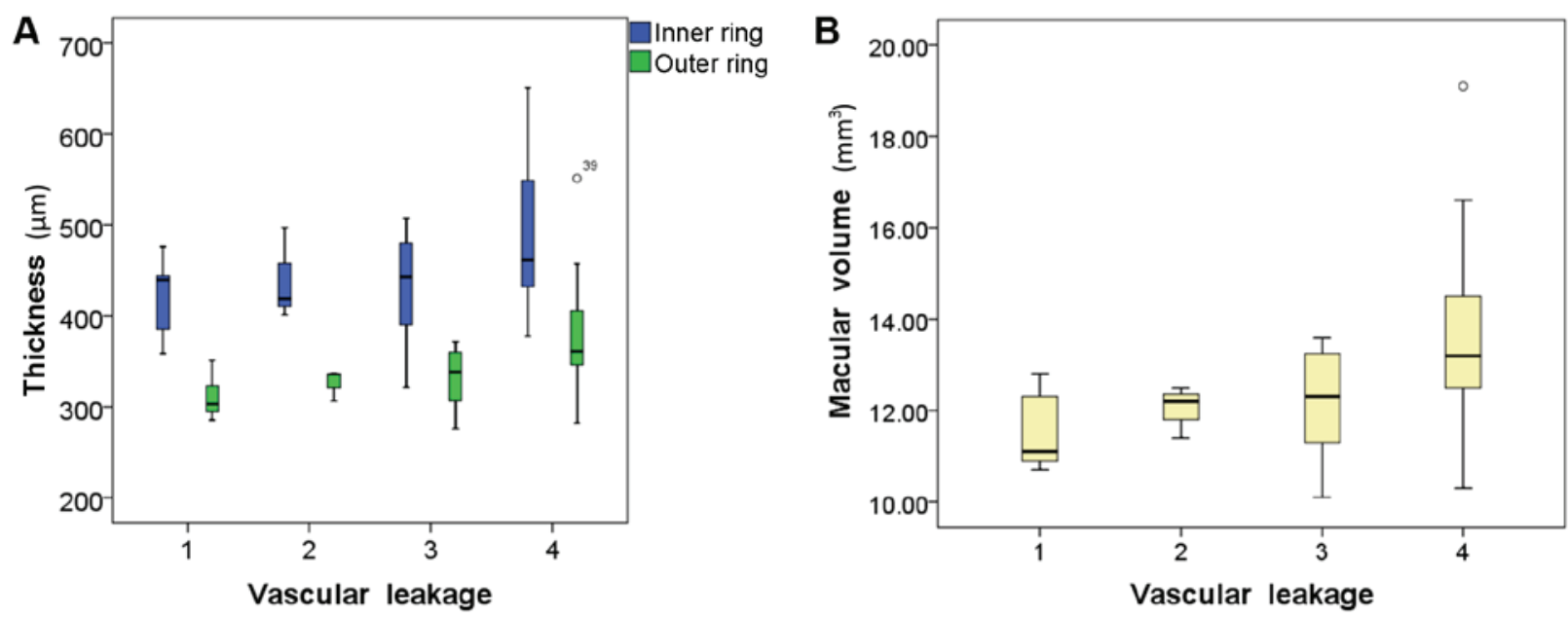

Figure 7. Macular vascular leakage is positively correlated with macular thickness and volume. Macular vascular leakage (measured by fundus fluorescein angiography) was positively correlated with (A) inner and outer ring thickness, and (B) macular volume (measured by optical coherence tomography).

contribution of increased vascular permeability to the CMT was not deemed to be significant, suggesting that vascular leakage has a limited effect on the central zone compared with the forces of traction caused by proliferative membrane contraction. A possible explanation for this is that the leakage of the retinal vasculature is affected by factors other than vascular distortion, including inflammation and the proliferative membrane itself (13). Visual acuity was determined as 

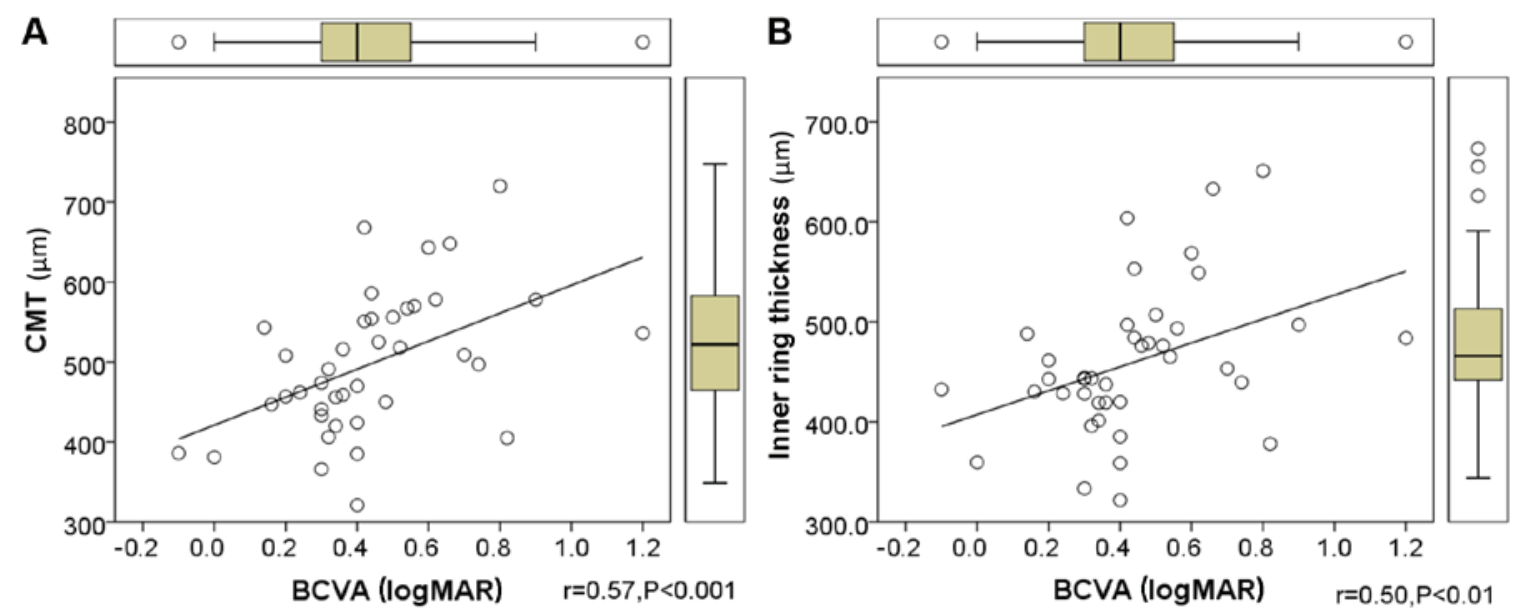

Figure 8. BCVA is positively correlated with thicknesses of the central zone and inner ring. BCVA scores positively correlated with (A) CMT and (B) inner ring thickness (measured by optical coherence tomography). CMT, central macular thickness; BCVA, best-corrected visual acuity; logMAR, logarithmically transformed score of the minimal angle of resolution.

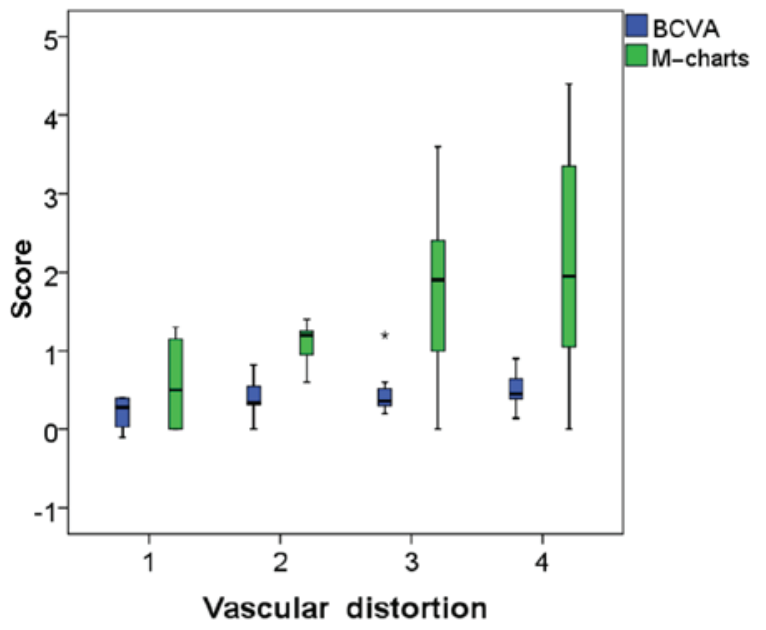

Figure 9. Macular vascular distortion is positively correlated with BCVA and M-chart scores. Macular vascular distortion was measured by fundus fluorescein angiography. BCVA, best-corrected visual acuity. "Extreme outlier.

BCVA in the present study and was positively correlated with the thickness of the central zone and inner ring. However, no correlations were identified among BCVA and outer ring thickness and macular volume. These results were consistent with those of previous studies (14-19). Additionally, the BCVA results were positively correlated with the grade of vascular distortion. The results of the present study were in accordance with those of a previous study by Gass (20), which stated that the loss of BCVA in eyes with ERMs was associated with distortion of the retina. It is important to note that visual acuity and metamorphopsia are subjective perceptual measurements, which may be affected by a number of factors in addition to the variables measured in the current study (21-23).

The results of FFA in the present study revealed that the degree of metamorphopsia was correlated with the grade of vascular distortion. These results are consistent with the results of a study by Arimura et al (24), which demonstrated that metamorphopsia scores measured by the M-charts were positively correlated with retinal contraction due to ERMs.
However, it is important to note that vascular distortion is just one factor affecting the degree of metamorphopsia. Other contributing factors, including the disorder of arrangement and edema of inner nuclear layer $(25,26)$, were not investigated in the present study.

OCT measures the exact retinal thickness in different macular regions and the whole macular volume. In the present study, a positive correlation between CMT, inner ring macular thickness and BCVA was determined, indicating that a thicker centralized area worsens visual acuity. These results are in accordance with those of previous studies $(17,19)$. However, there was no correlation between the OCT results and the severity of metamorphopsia. OCT does not directly identify the vascular changes that occur as a result of ERMs, including vascular leakage and distortion.

FFA is a commonly used tool in retinal vascular disease and macular edema diagnosis, as it identifies the severity of vascular leakage and distortion. In the present study, the association between the semi-quantitative variables of macular vascular changes and visual functions were analyzed. The grading of vascular distortion was positively correlated with BCVA $\log$ MAR and M-chart scores, demonstrating that changes to the macular vasculature are important in visual functions, particularly in metamorphopsia. This feature of FFA compensates for the limitations of OCT. The combination of OCT and FFA may be important as it may improve understanding of the pathophysiology and evaluation of visual functions in patients with ERM.

In conclusion, FFA may be an effective method of examining the vascular changes that occur in patients with ERMs. The identification of the correlation between FFA findings, OCT measurements and visual functions may improve understanding of the pathophysiological mechanisms underlying ERM development. FFA may be used to investigate the factors affecting the anatomical and functional prognosis of patients with ERM and estimate the severity of ERM development. FFA may also be adopted to determine the indication and timing of ERM surgery for the best prognosis, by comparing the severity of change in affected eyes prior to and following surgery. 


\section{References}

1. Pearlstone AD: The incidence of idiopathic preretinal macular gliosis. Ann Ophthalmol 17: 378-380, 1985.

2. Michels RG: A clinical and histopathologic study of epiretinal membranes affecting the macula and removed by vitreous surgery. Trans Am Ophthalmol Soc 80: 580-656, 1982.

3. Schmitz-Valckenberg S, Holz FG, Bird AC and Spaide RF: Fundus autofluorescence imaging: Review and perspectives. Retina 28 : 385-409, 2008.

4. Dell'omo R, Cifariello F, Dell'omo E, De Lena A, Di Iorio R, Filippelli $\mathrm{M}$ and Costagliola C: Influence of retinal vessel printings on metamorphopsia and retinal architectural abnormalities in eyes with idiopathic macular epiretinal membrane. Invest Ophthalmol Vis Sci 54: 7803-7811, 2013.

5. Niwa T, Terasaki H, Kondo M, Piao CH, Suzuki T and Miyake Y: Function and morphology of macula before and after removal of idiopathic epiretinal membrane. Invest Ophthalmol Vis Sci 44: 1652-1656, 2003.

6. Weinberger D, Stiebel-Kalish H, Priel E, Barash D, Axer-Siegel R and Yassur Y: Digital red-free photography for the evaluation of retinal blood vessel displacement in epiretinal membrane. Ophthalmology 106: 1380-1383, 1999.

7. Klein BR, Hiner CJ, Glaser BM, Murphy RP, Sjaarda RN and Thompson JT: Fundus photographic and fluorescein angiographic characteristics of pseudoholes of the macula in eyes with epiretinal membranes. Ophthalmology 102: 768-774, 1995.

8. Kadonosono K, Itoh N, Nomura E and Ohno S: Capillary blood flow velocity in patients with idiopathic epiretinal membranes. Retina 19: 536-539, 1999.

9. Maguire AM, Margherio RR and Dmuchowski C: Preoperative fluorescein angiographic features of surgically removed idiopathic epiretinal membranes. Retina 14: 411-416, 1994.

10. Bailey IL and Lovie-Kitchin JE: Vision acuity testing. From the laboratory to the clinic. Vision Res 90: 2-9, 2013.

11. Matsumoto C, Arimura E, Okuyama S, Takada S, Hashimoto $S$ and Shimomura Y: Quantification of metamorphopsia in patients with epiretinal membranes. Invest Ophthalmol Vis Sci 44: 4012-4016, 2003.

12. Arimura E, Matsumoto C, Nomoto H, Hashimoto S, Takada S, OkuyamaS and Shimomura Y: Correlations between M-CHARTS and PHP findings and subjective perception of metamorphopsia in patients with macular diseases. Invest Ophthalmol Vis Sci 52 $128-135,2011$

13. ZhaoF, Gandorfer A, Haritoglou C, Scheler R, Schaumberger MM, Kampik A and Schumann RG: Epiretinal cell proliferation in macular pucker and vitreomacular traction syndrome: Analysis of flat-mounted internal limiting membrane specimens. Retina 33 77-88, 2013.
14. Wilkins JR, Puliafito CA, Hee MR, Duker JS, Reichel E, Coker JG, Schuman JS, Swanson EA and Fujimoto JG: Characterization of epiretinal membranes using optical coherence tomography. Ophthalmology 103: 2142-2151, 1996.

15. Massin P, Allouch C, Haouchine B, Metge F, Paques M, Tangui L, Erginay A and Gaudric A: Optical coherence tomography of idiopathic macular epiretinal membranes before and after surgery. Am J Ophthalmol 130: 732-739, 2000

16. Arichika S, Hangai M and Yoshimura N: Correlation between thickening of the inner and outer retina and visual acuity in patients with epiretinal membrane. Retina 30: 503-508, 2010.

17. Michalewski J, Michalewska Z, Cisiecki S and Nawrocki J: Morphologically functional correlations of macular pathology connected with epiretinal membrane formation in spectral optical coherence tomography (SOCT). Graefes Arch Clin Exp Ophthalmol 245: 1623-1631, 2007.

18. Theodossiadis PG, Grigoropoulos VG, Kyriaki T, Emfietzoglou J, Vergados J, Nikolaidis P and Theodossiadis GP: Evolution of idiopathic epiretinal membrane studied by optical coherence tomography. Eur J Ophthalmol 18: 980-988, 2008.

19. Pilli S, Lim P, Zawadzki RJ, Choi SS, Werner JS and Park SS: Fourier-domain optical coherence tomography of eyes with idiopathic epiretinal membrane: Correlation between macular morphology and visual function. Eye (Lond) 25: 775-783, 2011

20. Gass JDM: Stereoscopic Atlas Of Macular Diseases: Diagnosis And Treatment. 3rd edition. CV Mosby, St. Louis, MO, 1997.

21. Falkner-Radler CI, Glittenberg C, Hagen S, Benesch T and Binder S: Spectral-domain optical coherence tomography for monitoring epiretinal membrane surgery. Ophthalmology 117: 798-805, 2010.

22. Inoue M, Morita S, Watanabe Y, Kaneko T, Yamane S, Kobayashi S, Arakawa A and Kadonosono K: Inner segment/outer segment junction assessed by spectral-domain optical coherence tomography in patients with idiopathic epiretinal membrane. Am J Ophthalmol 150: 834-839, 2010.

23. Shiono A, Kogo J, Klose G, Takeda H, Ueno H, Tokuda N, Inoue J, Matsuzawa A, Kayama N, Ueno S and Takagi H: Photoreceptor outer segment length: A prognostic factor for idiopathic epiretinal membrane surgery. Ophthalmology 120: 788-794, 2013.

24. Arimura E, Matsumoto C, Okuyama S, Takada S, Hashimoto S and Shimomura Y: Retinal contraction and metamorphopsia scores in eyes with idiopathic epiretinal membrane. Invest Ophthalmol Vis Sci 46: 2961-2966, 2005.

25. Okamoto F, Sugiura Y, Okamoto Y, Hiraoka T and Oshika T: Associations between metamorphopsia and foveal microstructure in patients with epiretinal membrane. Invest Ophthalmol Vis Sci 53: 6770-6775, 2012.

26. Watanabe A, Arimoto S and Nishi O: Correlation between metamorphopsia and epiretinal membrane optical coherence tomography findings. Ophthalmology 116: 1788-1793, 2009. 